\title{
Cathode Position Response of Large-Area Photomultipliers Under a Magnetic Field
}

\author{
T. Koblesky, J. Roloff, C. Polly, and J.C. Peng \\ Department of Physics, University of Illinois at Urbana-Champaign, Urbana, \\ Illinois 61801, USA
}

\begin{abstract}
With the increasingly common use of large area PMTs (photomultiplier tubes) for nuclear and particle physics experiments, information on the position dependent magnetic field effects across the PMT's photocathode is important to effectively analyze and understand data collected from PMTs. Using an automated twodimensional scanner, we have measured the PMT response to an external magnetic field as a function of the cathode position impinged by a collimated light source. This study shows a clear dependence on the cathode position in both collection efficiency and gain of the PMT in the presence of a magnetic field. In particular, a pronounced valley in the collection efficiency is observed for certain locations of the cathode when the magnetic field is transverse to the PMT axis. The pattern of the position dependence is presented for several different magnitudes and orientations of the magnetic field relative to the PMT.
\end{abstract}

\section{Introduction}

Large diameter hemispheric PMTs have been used extensively in many recent neutrino experiments including Superkamiokande [1], Kamland [2], SNO [3], MiniBoone [4, Icecube [5], and Chooz [6]. Ongoing experiments at DoubleChooz [7], DayaBay [8], and Reno [9], all aiming at precision measurements of the yet unknown neutrino mixing angle $\theta_{13}$ using neutrinos from nuclear reactors, also utilize 8-inch or 10-inch diameter hemispheric PMTs for neutrino detection. In these precision neutrino oscillation experiments, the value of the $\theta_{13}$ mixing angle will be extracted from a precise comparison of the number of neutrino events measured at near versus far sites. A good understanding of the performance of the PMTs is crucial for minimizing the systematic uncertainties of these experiments.

In this article, we report results from a measurement of the response of an 8-inch diameter hemispheric PMT in the presence of a magnetic field. The 
relatively large distance between the photo-cathode and the first dynode makes a large diameter hemispheric PMT more prone to the influence of ambient magnetic field, which would alter the trajectories of photoelectrons emitted from the photo-cathode. As a result, the PMT efficiency, as well as the PMT gain, could be significantly affected by the presence of the magnetic field.

Several studies have already been reported in the literature on the overall response of the PMT to magnetic field when light is shined uniformly over the surface of the photo-cathode [511,10]. However, the dependence of the PMT response as a function of the cathode position in the presence of a magnetic field has not yet been reported.

The goal of this study is to obtain information on the cathode position dependence of the PMT response as a function of the magnitude and orientation of the external magnetic field. This information could be used in a detailed modeling of the PMT response to magnetic field for analyzing the data, and could also help optimizing the design of future experiments utilizing large diameter hemispheric PMTs.

\section{Experimental Setup}

Figure1 shows the photo and schematics of the experimental setup. The PMT used in this study is an 8-inch Hamamastu R5912 [13]. Similar models of PMTs have been used in several neutrino experiments including LSND, MiniBoone, and SNO, and will also be used in DayaBay. For this study, a positive high voltage was applied to the PMT's anode. The PMT signal comes out of anode via the high voltage cable. In order to extract the signal, a splitter box was used to separate the high frequency components from the dc high voltage. The signal was fed into an ADC (analog-to-digital converter) to measure the integrated charge.

An LED, with a wavelength of $465 \mathrm{~nm}$, chosen for its high quantum efficiency for this PMT, was pulsed with a frequency of $500 \mathrm{~Hz}$. Although the source is fairly stable, the LED's light amplitude fluctuates slightly over the duration of a scan of the PMT. To correct for these fluctuations, three optical fibers diverted some of the LED light to a 2 inch diameter reference PMT shielded from magnetic fields in $\mu$-metal, while the remaining four optical fibers were directed towards the 8-inch PMT. The intensity of the LED can be monitored by the response of the reference PMT. The variation throughout the scan was minimal; in a 12 hour scan, the reference PMT recorded only a $10^{-3}$ variation of LED intensity. This small amount of fluctuation was removed in the analysis by dividing PMT's response by the reference PMT's response. 

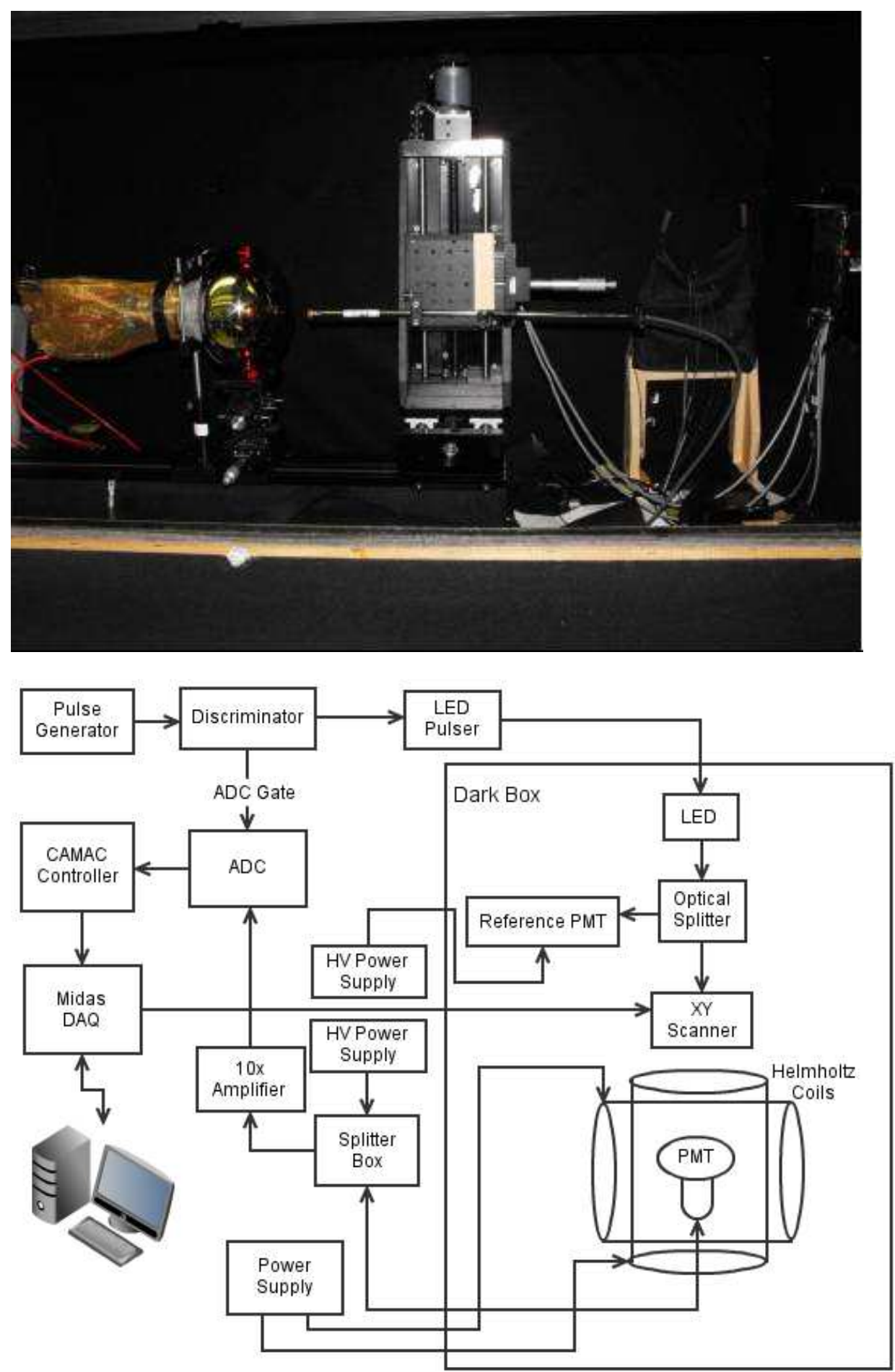

Fig. 1. a) A photo showing the PMT and the scanner in the dark box. b) The schematics of the setup of the experiment. 


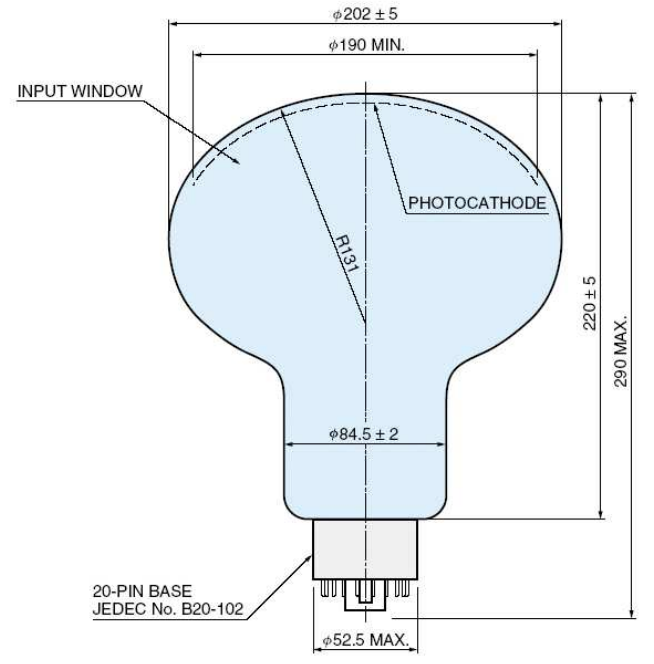

PMT Orientation in Dark Box

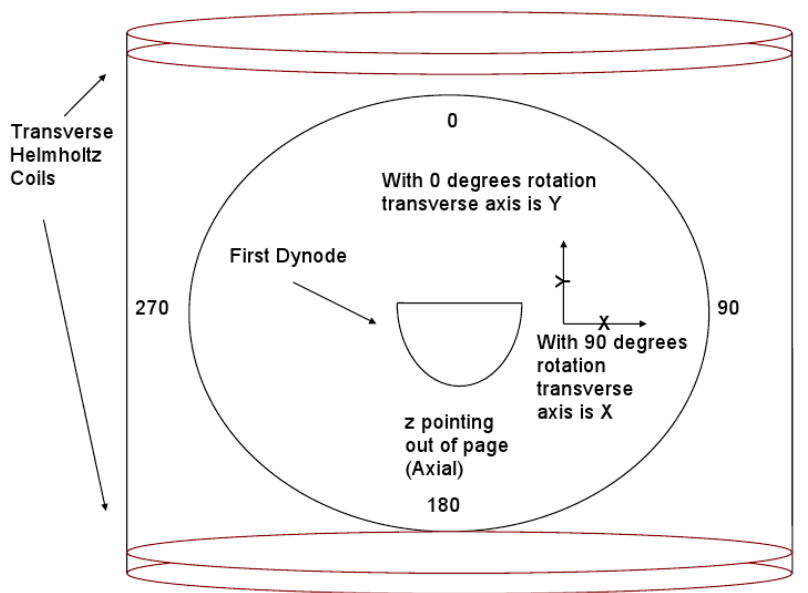

Fig. 2. a) Side-view of the Hamamatsu R5912 PMT. b) End-view of the PMT. The $\mathrm{x}-\mathrm{y}$ plane is also indicated

In order to aim the fiber at various positions of the photo-cathode, an automated 2D scanner was used. This scanner consisted of a platform which could be moved to various $(\mathrm{x}, \mathrm{y})$ positions within range by stepping motors in two orthogonal directions. The $\mathrm{x}-\mathrm{y}$ plane is perpendicular to the axis of the PMT, which is along the z-axis. The scanner has a range of $20 \mathrm{~cm}$ in both directions perpendicular to the z-axis with a minimum step size of $1 \mu \mathrm{m}$ [12. The PMT and optical fibers were oriented as shown in Figure 1, and the fibers were positioned on the scanner so that it would move up, down, left, and right to aim at various points on the PMT photo-cathode. The fibers were positioned 2 inches away from the photo-cathode, with an average circular spot size of 2 $\mathrm{mm}$.

Two pairs of 45-cm diameter Helmholtz coils were positioned around the PMT in order to cancel the earth's magnetic field or to induce magnetic fields of a given magnitude and direction. The first pair of Helmholtz coils was positioned along the z-axis, and the second along the y-axis. The two pairs of Helmholtz coils served also to cancel the earth's field of -0.47 gauss in the y-direction and 0.2 gauss in the z-direction. The earth's magnetic field along the x-axis was negligible. By inducing magnetic fields with these Helmholtz coils we were able to create magnetic fields within a range of magnitude in any direction in the $\mathrm{y}-\mathrm{z}$ plane.

A Lecroy 2249W ADC was used to digitize the charges collected from the R5912 and the reference PMT. The LED pulser triggered a discriminator that provided an ADC gate width of 55 ns. This width suitably contains the PMT signal which had a width on the order of $30 \mathrm{~ns}$. The Midas DAQ system which interfaced with the CAMAC SCSI Bus Crate Controller(73A) was used to record data from the $\mathrm{ADC}$. 


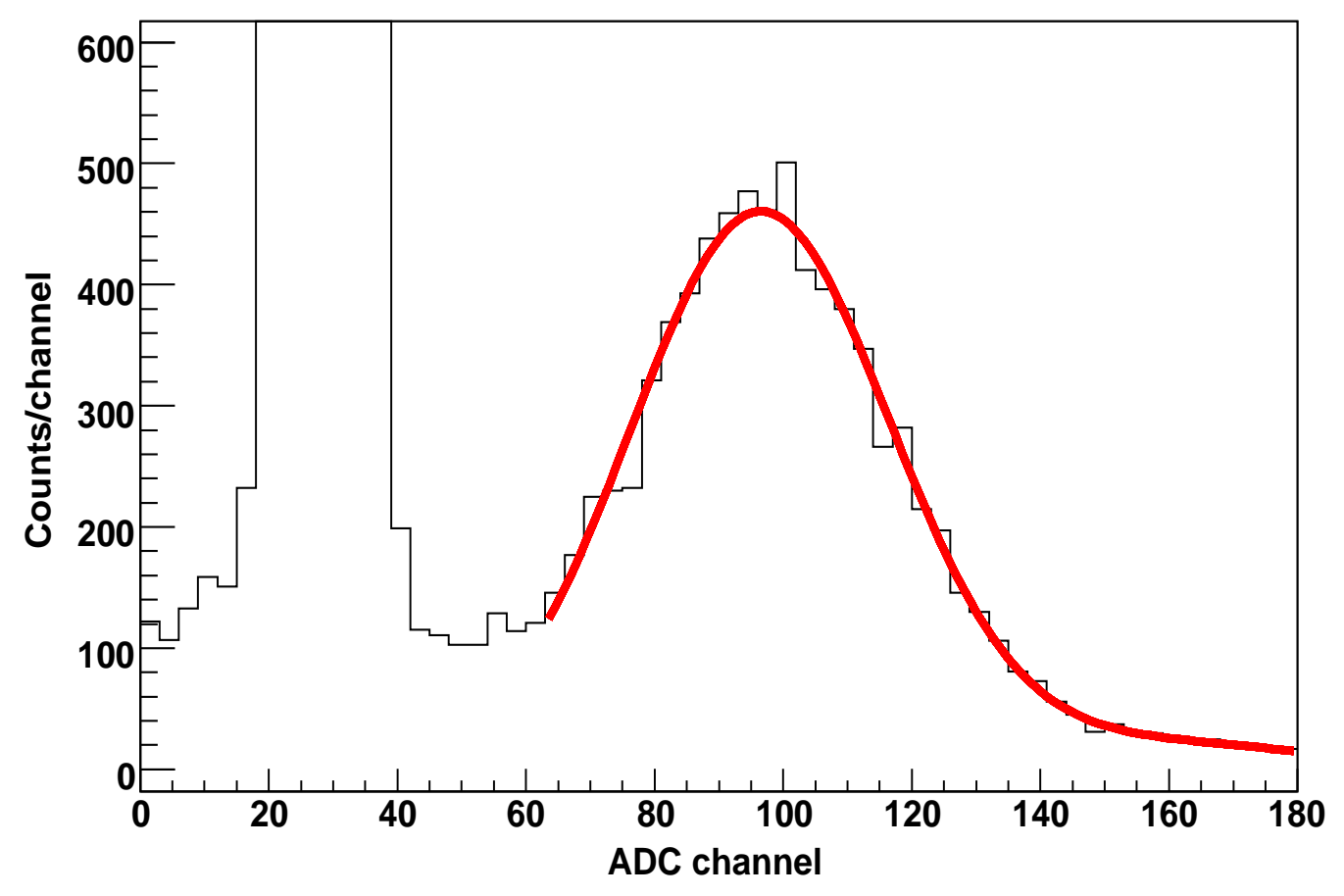

Fig. 3. A typical ADC spectrum showing the SPE and the pedestal peak.

\section{Results of Measurements}

The effects of magnetic fields on the PMT collection efficiency and gain were measured at different orientations of the magnetic field with respect to the PMT. The collection efficiency of a PMT refers to the probability of electrons emitted by the photo-cathode reaching the first dynode. The region between the photo-cathode and the first dynode is most susceptible to magnetic field effects. As shown in Figure 2b, the first dynode is not azimuthally symmetric. Hence the collection efficiency is in general not azimuthally symmetric with respect to the cathod position from which photo-electrons are emitted. This asymmetry can be further enhanced in the presence of magnetic field.

To determine the collection efficiency and gain, we adjusted the LED intensity so that the single-photoelectron (SPE) peak is the dominant feature in the ADC spectrum. A typical ADC spectrum is shown in Figure 3, The location of the SPE peak is fitted with a Gaussian distribution. After subtraction the pedestal channel from the SPE channel, the PMT gain is calculated taking into account the ADC calibration $(0.25 \mathrm{pC} / \mathrm{ch})$ and the effect of the terminator in the PMT base circuit. The high voltage was set at a PMT gain of $\sim 10^{7}$.

ADC spectra, similar to the one shown in Figure 3, were also analyzed to extract information on the PMT collection efficiency. For a fixed LED in- 

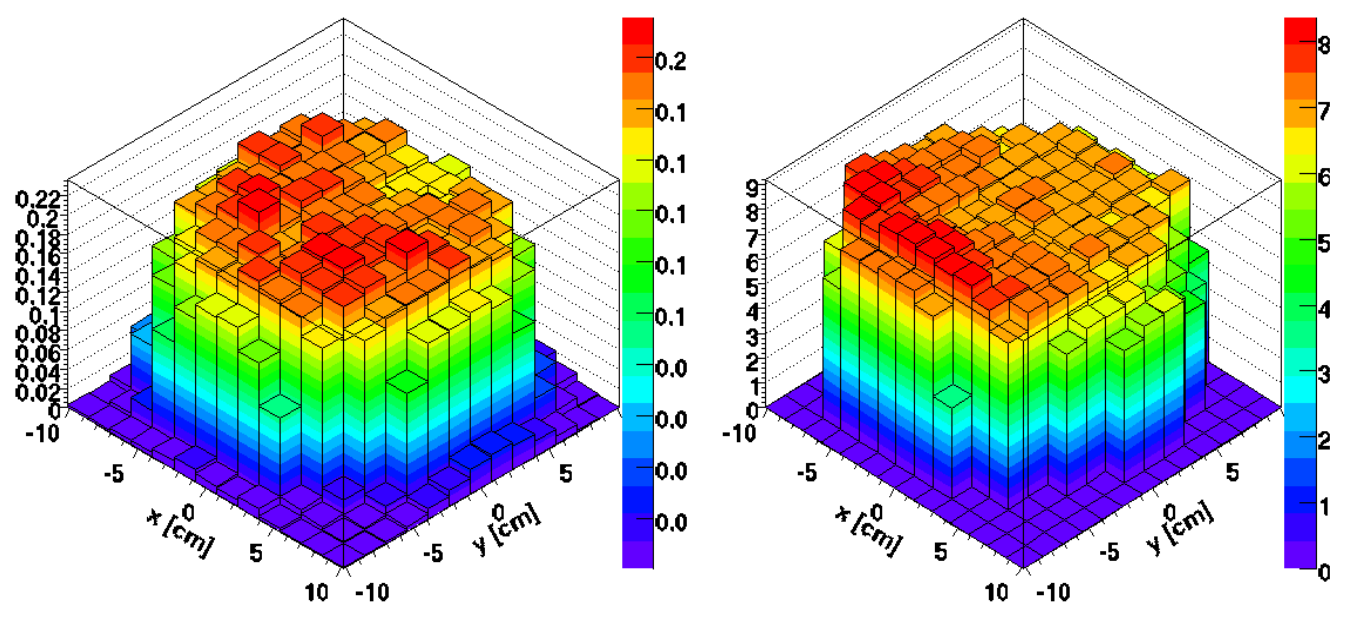

Fig. 4. a) Mean value of the photoelectron, $\mu$ and b) Gain of the PMT in units of $\log$, of the PMT in zero magnetic field as a function of photo-cathode position.

tensity, the PMT detection efficiency is proportional to the mean value of photoelectrons, $\mu$, measured by the PMT. Using the Poisson distribution, $P(k)=\mu^{k} e^{-\mu} / k$ !, to describe the probability of detecting $k$ photoelectrons, one can readily calculate $\mu$ from $P(0)=e^{-\mu}$. The probability of detecting no photoelectron, $P(0)$ is determined from the number of pedestal events divided by the total number of events.

To measure the PMT response to magnetic fields, we first measured its performance in zero magnetic field. The earth's field ( -0.47 gauss in the y-direction and 0.2 gauss in the z-direction) was cancelled by the two sets of Helmholtz coils producing opposite magnetic fields. Figure 4 a shows that $\mu$ is rather uniform across the cathode surface except near the edge, where reflection of LED light from the glass surface due to a larger incident angle led to lower collection efficiency. Figure $4 \mathrm{~b}$ shows that the PMT gain is largely independent of the cathode position at zero magnetic field. Note that Fig. 4 is obtained by analyzing a large number of ADC spectra collected for various grid positions of the scanner.

The response of the PMT to a net external magnetic field has been measured with the magnetic field pointing perpendicular or parallel to the axis of the PMT. Figure 5 shows the PMT's relative collection efficiency $\epsilon=\mu / \mu_{0}$, when the net magnetic field is -0.5 gauss in the $y$-direction. The relative collection efficiency is the rate of $\mu$ measured at a given magnetic field over the corresponding $\mu_{0}$ measured at zero magnetic field. Figure 5 a shows that the presence of a magnetic field significantly modifies the PMT collection efficiency, and a striking position dependence is observed for $\epsilon$. A pronounced valley in $\epsilon$ centered around $x=4 \mathrm{~cm}$ and $y=0 \mathrm{~cm}$ is observed. 

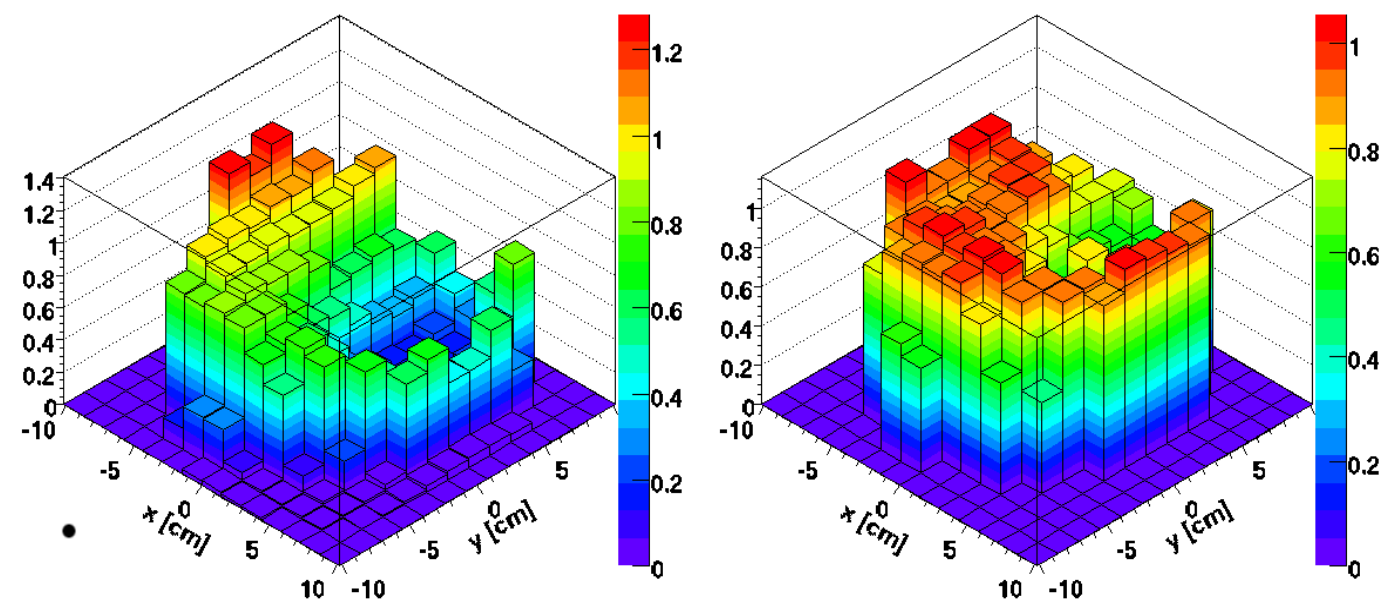

Fig. 5. Plots of a) The collection efficiency of the PMT b) The gain of the PMT with a -0.5 gauss magnetic field in the $\mathrm{y}$-direction.
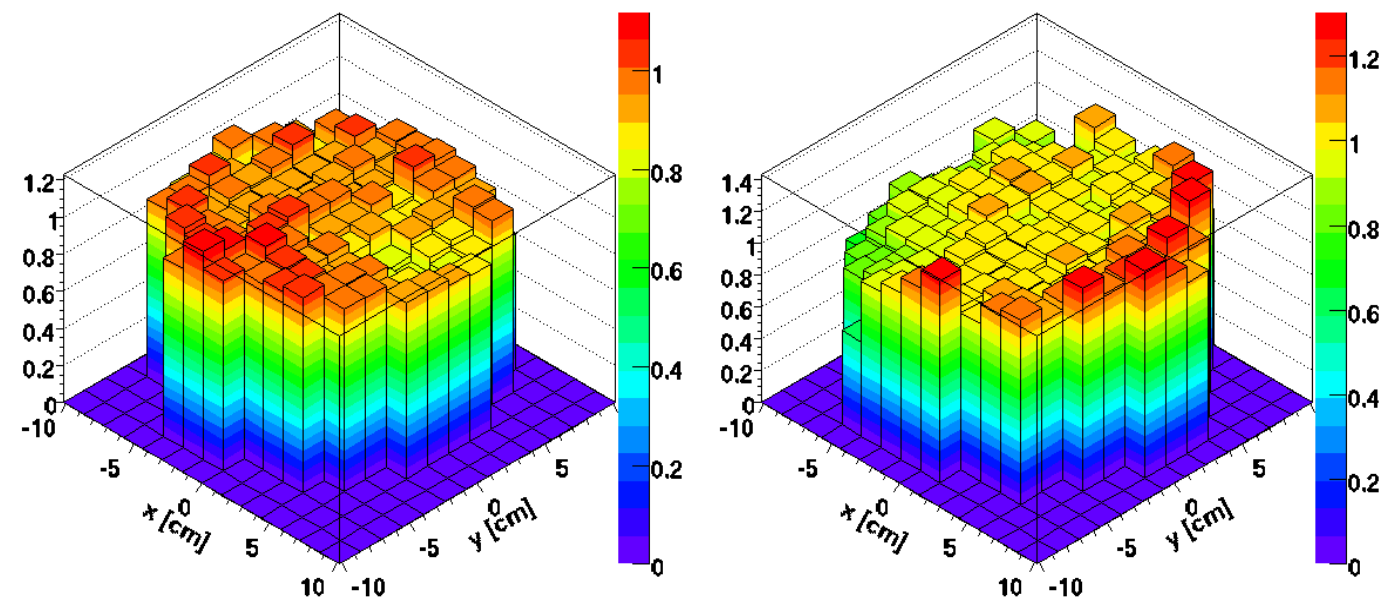

Fig. 6. Plots of a) The relative collection efficiency of the PMT b) The relative gain of the PMT for several points on the photocathode with a -0.5 gauss magnetic filed in the y-direction while the PMT is rotated by $90^{\circ}$.

Figure 5b shows the PMT relative gain, $g=G / G_{0}$ as a function of the cathode position. $G$ is the PMT gain measured at a non-zero magnetic field, and $G_{0}$ is at zero magnetic field. As shown in Fig. 5, the effect of the magnetic field on PMT gain is much smaller than on the PMT collection efficiency. Nevertheless, $g$ also depends on the cathode position and has a less pronounced valley at the same region as that of $\epsilon$. The observed cathode position dependence of the PMT gain implies that the mean deviation of the PMT gain would be larger in the presence of a magnetic field.

Figure 6 shows the PMT response when the PMT is rotated by $90^{\circ}$ around 

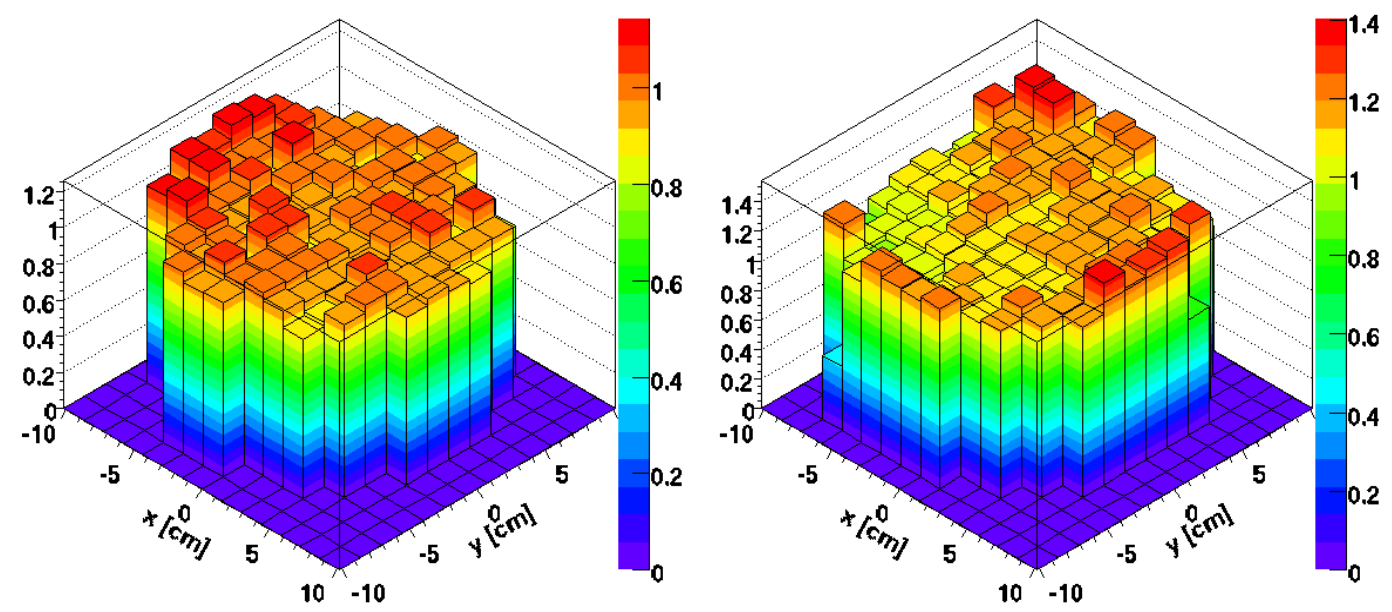

Fig. 7. Plots of a) The collection efficiency and b) The gain of the PMT with a -1 gauss magnetic field along the axis of the PMT.

its axis. There is a notable difference between the response of the PMT in Figure 5 and Figure 6 when considering that the only difference between the plots is that the magnetic field has been rotated by $90^{\circ}$. The pronounced hole that appears in the collection efficiency $\epsilon$ of Figure 5 a disappears when the transverse magnetic field is rotated by $90^{\circ}$ as seen in Figure 6a.

When an axial magnetic field is induced, this dip is not present, and the response remains relatively stable across the photocathode (Figure 7). Since a magnetic field in the axial direction would not significantly bend the electrons away from the first dynode, the changes in the response of the PMT are expected to be much less noticeable. Figure 7 confirms that even in the presence of a 1 gauss magnetic field, the amount of decrease in both collection efficiency and gain is minimized with an axial magnetic field.

\section{Conclusion}

We have measured the PMT response to various orientations of external magnetic fields as a function of the cathode position impinged by a collimated light source. A clear dependence on the cathode position in both collection efficiency and gain of the PMT is observed. Although the effects on the PMT gain is smaller than that on the PMT collection efficiency, both effects have been observed. In particular, a pronounced valley in the collection efficiency is observed for certain locations of the cathode when the magnetic field is transverse to the PMT axis. An axial magnetic field, however, has been shown to create a relatively small change in collection efficiency and gain. This study shows that a detailed modelling of the response of a large diameter PMT in the 
presence of an external magnetic field should take into account the significant cathode position dependence.

\section{Acknowledgements}

We gratefully acknowledge the advice and assistance of Professors D. Hertzog and K.B. Luk during the course of this measurement. This work is supported by the U.S. National Science Foundation. 


\section{References}

[1] S. Fukuda et al., Nucl. Instrum. Meth. A 501, 418 (2003).

[2] K. Eguchi et al., Phys. Rev. Lett. 90, 021802 (2002).

[3] J. Boger et al., Nucl. Instrum. Meth. A 449, 172 (2000).

[4] A. Aguilar-Arevalo et al., Nucl. Instrum. Meth. A 599, 28 (2009).

[5] R. Abbasi et al., Nucl. Instrum. Meth. A 618, 139 (2010).

[6] M. Appollonio et al., Eur. Phys. J. C 27, 331 (2003).

[7] F. Ardellier et al., http://arxiv.org/abs/hep-ex/0606025.

[8] X. Guo et al., http://arxiv.org/abs/hep-ex/0701029.

[9] J. K. Ahn et al., http://arxiv.org/abs/hep-ex/1003.1391.

[10] E. Calvo et al., Nucl. Instrum. Meth. A 621, 222 (2010).

[11] S. J. Brice et al., Nucl. Instrum. Meth. A 562, 97 (2005).

[12] D.A. Simon et al., NIM A335 (1993) 86-101.

[13] http://www.hamamatsu.com 